l'existence de ce maximum ; les proportions de pluie ou de neige auront besoin d'être plus suivies ; l'étude des variations thermiques, aux différents étages de la montagne, sera entreprise, pendant la belle saison, cette année même.

Il ne serait pas moins intéressant de rechercher, au moyen de baromètres enregistreur's, jusqu'a quel niveau se font sentir' les mouvements tourbillonnaires de l'atmosphère, si violents dans les régions supérieures et qui sont déjà atténués à la hauteur de Tête-Rousse, mais nous n'avons pas les instruments nécessair'es, non plus que les hvgromètres enr'egistreurs.

Il appartiendra à l'Administration des Eaux et Forêts, qui a déjà tant fait, comme vous venє\% de le voir, pour favoriser los observations en haute montagne, de dire si elle veut étendre encore, ce qui est tr'ès souhailable, le champ de ses investigations et entreprendre ces nouvelles études.

\section{Le Reboisement en Savoie}

Communication faitc par M. Mougrn, Inspecteur des Eaux et Forèts, au Congrès de Lyon de l'Associalion francaise pour l'avancement des Sciences, au cours de la séance du 3 août 1906.

On ne discule plus l'opportunité du reboisement en mon. tagne. Lo retour périodicue des inondations de la Loire et surtoub du. Rhône et de ses affluents ont misi celte question à l'ordrte du jour". Depuis longtemps, on a signalé la dégr'alation progr"essive des Alpes, les ravages de leurs torrents et la répercussion fàcheuse de la dénudation des pentes sur le l"égime des eaux el la slabilité du lit des rivières. Administrateur's, ingénieurs el lorestiers étudièrent les causes des débordements, des érosions des versants, de l'enlisement des villages et des cultures.

Ein 1841, Surell, ingénieur des Ponts et Chausséess du départiement des Hautes-Alpes, dans un livre qui fait autorité, a démontrél les quatre proposilions. suivantes :

$1^{\circ}$ La présence d'une fortêt sur un sol empèche la formation des torrents ;

$2^{\circ}$ Le débcisement d'une forèt livre le sol en proie aux torrents :

$3^{\circ}$ Le dévelcppement des for'êts provoque l'extinction des torrents ;

$4^{\circ}$ La chute des forêts redouble la violence des torrents, peut mème les faire renaître.

Dans son "Eitude sur les travaux de reboisement et de gazonnement des monlagnes ", parue en 1878, un forestier, Demontzey, en exposant les mo'vens pratiques de rétablir la forêt, là où elle avait đisparu, écrivait :

"Il est indispensable de bien préciser le but qu'on se propose en entreprenant une semblable opération. Ce but est la création d'une végétation ligneuse qui répond aux conditions suivantes :

'" $1^{\circ}$ Posséder des racines assez puissantes, pour enserr'er le sol dans leurs innombrables réseaux, le rendre au besoin plus perméable et le priotégter contre l'entraînement.

" $2^{\circ}$ Présenler" un couvert assez complet pour abriter la surface contre les influences météorologiques;

" $3^{\circ}$ Fourmir" un humus de plus en plus abondant, appelé, d'une part, à fertiliser le sol et à augmenter la puis'sance de la végétation et, d'autre part, ì favor'iser le ralentissement et la régularisation du débit des eaux pluviales ou des neiges fondant à sa surface ;

" $4^{\circ}$ Maintenir sans interruptions momentanées et perpétuellement, ces salutair'es effets et les développer avec l'aide du temps.

"La futaie, seule, est capable de satisfaire, sans restrictions, à l'ensemble de ces conditions ; c'est donc sa création et son développement qui doivent ètre le but final du reboisement $n$.
Depuis l'époque où lurent écrites ces lignes, on arriva a démontrer, par des séries d'observations, que le couvert des résineux arrêtait environ la moitié de la lame d'eau tombée ; que l'humus absorbait jusqu'à deux fois et demie son poidis des eaux atmosphérriques qui lui parrvenaient et qu'enfin les racines faisaient baisser la nappe phréatique plus bas sous bois que hor's bois, assainissant, asséchant ainsi la conche supérieur'e du sol. Pair respiration, les arbres renvoient dans l'atmosphèr' $€$. une partie de cette humidité, d'où un climat plus égal, des pluies plus fréquentes.

Enfin la présence de rideaux boisés brise la violence des vents qui dessèchent les vallées comme la Maurienne ou qui descendent en rafales puissantes des cols élevés des Alpes, du P'elit-Saint-Bernard, par exemple.

Mais alor's qu'on avail attaqué énergiquement le phénomène torrentiel dans les Hautes et les Basses-Alpes, dans l'Isère, dans la Dr'òme, il semblait qu'on eût oublié les départements de la Savoie. Pourtant, immédiatement avant et après l'annexion de 1860, des inondations, des laves (on appelle amsi des coulées boueuses produites par. les torrents), avaient causé dans la région de terribles dommiages el avaient eu leur répler"cussion sur l'Isère et sur' le Rhône lui-même.

Le $1^{\text {er }}$ novembre 1859 , c'est l'Isère, l'Arc, l'Arly qui débordent, rompent leurs digutes et détreminent la submersion du Grésivaudan et d'une partie de la ville de Grenoble.

En 1866 , le 25 septembre, c'est le tor'rent de l'Envers en Maurienne qui barre l'Ar'c el amène une débâcle formidable. "LArc, dit dans son rapport, le Ministre des Travaux Publics, a éprouvé une crue telle qu'on en a jamais signalé, même aux époques les plus reculées. Fn quelques heures, la vallée étail dévastée, la route impériale du Mont-Cenis clétruite sur plus de six kilomètres de longueur, etc..." ".

Un peu plus tard, le 20 juillet 1871 , c'est le ruisseau de Saint-Julien qui coupe encore la route nalionale de Paris à Turin et interrompt, pendant vingt-neuf jours, tout trafic sur la voie ferrée du Rhòne au Mont-Cenis. Une nouvelle lave du même tortrent ar"rête également la circulation pendant dix-neuf jours : du 24 juillet au 12 août 1872 .

En 1875, le 18 janvier, la ville de Chambéry est inondée par les eaux du torrent de Leysse.

Comme si ce n'était pas assez, des torrents nouveaux naissent, se développent. C'est la Grollaz, inoffensif ruisseau, qui vient recouvrir de matériaux la voie ferréie près de Saint-Michel-de-Maurienne ; c'est, en Tarentaise, le Sécheron qui, dès 1868, menace d'engloutir un des hameaux de la commune de Le Bois ; ciest le Nant-Trouble, à Ugines, de 1877 à 1882, qui char"rie dans la plaine des Corroies plus de 1 million de mètres cubes des terres arrachées à la montagne du Siapey et étale ses déjections jusque sur la chaussée de la route départementale d'Ugines à Seyssel, qui cependant était établie en remblai.

La lèpr"e torrentielle allait augmentant tous les jours, car la Savoie, jadis couverte de vastes forềts, se déboisait chaque jour davantage.

Dans le cours du XIX ${ }^{e}$ siècle, la Tarentaise voit disparâ̂tire 11,5 p. 100 de ses massifs communaux et la Maurienne 38 p. 100. Au pied du Mont-Cenis, le seul canton de Lanslebourg a perdu 41 p. 100 de sa surface forestière !

Il était dono excessivement urgent de réagir. En 1880, le service forestier commence la correction du torrent de la Grollaz, en Maurienne. Mais, devant l'opposition que rencontra le reboisement, il doit se borner aux travaux d'art, barrlages et drains destinés à consolider temporairement le sol.

Six ans plus tard, l'Etat acquiert le glissement de Sécheron, l€ draîne et exécute ses premières plantations. Ce n'est qu'en 1894, que l'Administration des Eaux et Forêts acquiert 1.294 hectar'es dans les vallées de l'Isère et de. l'Arly et 2.518 hectar'es dans celle de l'Arc, en amont de Saint-Jeande-Maurienne. Malheureusement, ces surfaces fort insuffl- 
santes se trouvent n'êtr'e que des berges de ravins dont le reboisement ne pourra agir, d'une façon efficace, sur le régimè des eaux. C'est tout ce que la loi du 4 avril 1882 sur la r'estauration des terrains en montagne per'met d'exproprier pour cause d'utilité publique.

On reste donc toujour's exposé à des crues brusques, violentes, considérables et, par suite, on a encore à redouter le r'etour, dan's les vallées principales, des grandes inondations qui les ont déjà désolées. Bienheureux encore si les' ouvrages de correction des torrents résistent au choc et aux érosions des eaux ! De leur résistance seule dépend, dans les conditions actuelles, le maintien, dans les gorges de la montagne de masses considérables de terre et de gravier".

Pouir" arriver à maintenir" d'une l'açon durable le sol sur les pentes, à ralentir $l \epsilon$ ruissellement et, par suite, à prévenir les affouillements, les charriages de matériaux et les débortdementsis, il fauldra nécessairement augmenter, en Savole, les surfaces reboisées. Commie les pérlimètres (c'egf ainsi que l'on nomme les terrains dont la restauration est déclarée d'utilité publique et qui sont acquis par l'Etat) se trouvent dans les situationsis les plus variées et à des altitudes aillant de 400 à 3.200 mètres, les travaux de reboisement déjà exécutéls donnent des indications précieuses pour l'avenirr quand il s'agir'a de créer de vastes peuplements.

La' première question qui se pose est naturellement celle-cil :

Quelle est la limite de la végétation forestière ?

D'apr'ès Demontzey, on doit reboiser le plus haut possible, " et ne s'arrêter qu'aux terrains où les neiges sont suscepti"bles de demeur'en" pendant plusieurs années de suite ". En d'autres termes, la limite supérieure de la végétation forestièr'e coïnciiderati avec la limite des neig'es éternelles.

or, il résulte d'observations précises, exécutées depuis 1901, au glacier de 'Tête-Rousse, à 3.200 mètr'es d'altitude, au S.-O. du massif du Mont-Blanc, que la fusion a fait disparaître toute la neige hivernale pendant la belle saison, sauf à la fin de 1902, où on a constaté l'existence d'un résidu neigeux.

La limite des neiges persistantes serait donc fort voisine de 3.200 mètríes. Ce résultat est confirmé par les modificar tions survenues dans les petits glaciers suspendus de toute la réfigion. Ainsi le glacier de Glière ou de Traversaille (altitude, 2.850. mètr'es), dans le massif des Aiguilles Rouges, n'existe plus depuis 1902.

Fin Tarentaise, la partie du glacier de Calabre qui partait du col de Rhème (3.602 mètresi) et descendant jusqu'à la cote 2.700, est entièrement fondue. Lie g:lacier de la vallée Etroite, qui atteignait 2.985 mètresi au-dessus du niveau de la mer, le gllacier qui s'étendait de la cime de Carron (3.149 mètres) au col de la Vallée Etroite (2.874 mètres), ont également disparu. En Maurienne, le glacier de la Belle-Plinier, situé sur des terrains périmètrés (3.091 mèlines), le glacier à l'Ouest de la cime du Grand-Vallon (3.134 mètres), se sont aussi évanouis; celui de Valmeinier" n'est plus qu'un simple névé.

Donc, dans toute la Savoie, la fusion l'emporte sur lalimentation au-dessous de la courbe de 3.200 mètres.

Aux environs de 1.860, au moment d'un maximum de glaciation, . la limite des neiges persistantes se trouvait à peu pir'ès à 2.700 mètres ; 1820 fut l'éproque du plus grand développement glaciaire du XIX siècle, et, avec quelque vraisemblance, on suppose que celte limite ne se trouvait pas alors à un niveau supérieur' à celui de 1860 .

On peut donc conclure de ces observations que la forêt n'a pras dépassé l'altitude de 2.700 mètres, mais a-t-elle pu l'atteindre? Il est difficile de le croir'e, car nulle part on n'a rencontr'é de souches aussi haul: A Valloire, à Saint Martin-de-Belleville, les tiges et les restes d'arbres ensevelis sous des graviers ou recouverts d'eau ne se sont pas trouvés à une cote aussi élevéle.
Voyons maintenant jusqu'où peut monter la végétation forestière. Au-dessus de Modane, sur le versant nord du Mont-Rond, dans des terrains domaniaux, un peu au-dessous de vicilles souches blanchies de Pin Cimbro ou Arole, on aperçoit des semis naturels de mème essence ; l'altitude pirise au baromètre s'est trouvée de 2.430 mètres. Il est très probable que cette r'égénération s'est faite depuis que l'Etal est devenu propriétaire du sol et que, par suite, le pâturage est interdit; le nombre des jeunes sujets, quoique restreint, n'est cependant pas assez r"éduit pour permetre de conclure à un cas fortuit. En rapprochant ce fait de réensemencement naturel du voisinage d'anciennes souches situées plus haut, on voit qu'on se tiendra bien près de la vérité, en adoptant, en Savoie, comme limite supérieure de la végétation forestière, la cote de 2.500 mètres.

Ce sera, là, la limile supérieure normale de la zone subalpine, telle que la définit M. le professeur Flahault.

Chacque espèce ligneuse a aussi une aire spécifique qui est fonction de l'altitude, de l'exposition, du climat et du sol, qu'il est indispensable de connaitre quand on veut exécuter des travaux de reboisement. Voici le résumé de nos observations en Savoie :

Le Sapin argenté, Abies pectincita. - Il est indifférent a la base géologique, mais est rare sul" les gypises et les argiles compactes. Il aime surtout les sols frais et profonds et les expositions N.-E. Atteint l'altitude maxima de 2.000 mètres (Maurienne).

L'Epicéa, Spicea excelsa. - $\Lambda$ les mèmes préférences que le Sapin. mais il se rencontre à toutes les expositions ; il arrive jusqu'à 2.300 mètr'es au-dessus du niveau de la mei (Modane) et descend jusqu'au fond des vallées.

Le Mélèze, Larix europea. - Croît aussi bien sur" tous les sols ; pourvu qu'ils soient meubles et profonds, il prospère. On le r'encontre à loutes les expositions et à des altitudes var'iant entre 2.380 (Modane) et 650 mètres (Villargondran). Ce résineux aime surtout la vive lumièr'e el réussit difficilement dansi les régions brumeuses comme le Chablais.

Le Pin Sylvestre, Pinus sylvestris. - Vit sur tous les terrains indistinctement, même sur les gypses, dans loutes les expositions, entre 2.000 mètres (Maurienne) et le fond des vallées. Pour reboisiar les pentes brûlées du soleil, les sols peu profonds, pierailleux ou à éléments minéraux stériles (ar'ènes, gypses), il rend les plusi girands services.

Le Pin de monlagne, Pinus montana. - Comme le Sylvestre, est très précieux pour le reboisement des terrainis' les plus rebelles, les plus secs, bien qu'il semble prérére les sols frais. Atteignant la cote 2.200 mèbres (Bozel), il ne descend gàere naturellement au-dessus de 900 mètr'es. Planté plus bas, à la différence de beaucoup d'arbres, son accroissement ne siemble pas plus rapide.

LiArole ou Pin Cembro, Pinus cembra. - Se trouve aussi sur tous les sols; mais il réussit surtout sur les terrains meubles et frais. Dépassant l'altitude de 2.100 mètres (Mo* dane), il est tout indigué pour les plantations en haute montagne, car' il résiste vigoureusement aux tempêtos, aux gelées et à la neige; dans la correction des couloirs d'avalanche, il esit indispensable. Sa limite inféricure est aux cnvirons de la courbe de 1.550 mètres.

Parmi les feuilles qui, dans les régions basses, peuvent entrer dans les massil's boisés que l'on veut créer, il con. vient de citer le Hêtre, Fargus sylralica, asse\% indifférént à la base geẻologique. Cet arbre craint les bas-fonds mar'écageux ou tourbeux et escalade les pentes jusqu'à 1.700 mètr'es d'altitude. A l'inverse du Mélèze, il exige un climat brumeux et, dans les grandes vallées de l'Aro, de l'Isère, do l'Arve, il ne dépasse guère, comme je l'ai maintes fois noté, les points où, en automne, s'arrêtent les brouillards. SaintMichel, Lime et les Houches marquent les limites du domaine du Hêtre en Maurienne, en Tarentaise et en Fauci- 
gny. Parl contre, le Chablais qu'inondent, en octobre et en novembre, les vapeurs du Léman, est le pays de prédilection de cette essence.

Le Chêne rouvre, Quercus robur. - Excellent pour fixer le sol par son puissant enracinement, pousse sur tous les terrains, les gypses et les cargueules exceptés ; il atteint 1.400 mètres d'altitude.

Le Bouleau, Bctula alba. - N'est pras exclusif au point de vue du sol ni de l'exposition; on le r'encontr'e jusqu'à 2.100 mètres (Saint-Julien-de-Maurienne). Il est recommandable par sa résistance au froid et aux intempéries.

LAulne blanc, Alnus incana. - Appelé Verne ; semble indifférent à la base géologique et l'exposition. Remontant les vallons jusqu'à 1.300 mètres d'altitude, de croissance rapide, il s'empar'e aisément des places de gravier", les protège, les couvre et les fertilise de ses feuilles mortes et permet ainsi l'introduction, en sous-élage, d'essences plus précieuses, Sapin ou Epiciéa. Nous ne citernons que pour mémoire le Frêne, qui pénètre jusqu'aux fond des vallées les pilus reculées (Bonneval-sur'-Arc); le Charme, peu abondant dans le département de la Savoie, le Ghâtaignier, les Erables, les Ormes, les Alisier's et Sorbiers qui ne sont pas des essences propres à for'mer" des peuplements complets, mais dont l'introduction diversifie heur'eusement les massifs en les mettant à l'abri d'une destruction totale, par suite d'une invasion d'insectes ou de champignon's.

Afin de se procurer lest graines for'estières nécessaires aux travaux de reboisement, I'Administration des Eaux $6 t$ Worêtis a créé en Savorie deux sécheries scollaires, l'une aux Fourneaux, près de Modane, et l'autre à Moùtiers. On n'y triaite que les cônes d'arbres résineux de la r'égion, Epicéa, Mélèze, Pin Sylvestre, Pin de montagne et Pin Cembro, recueillis dans les forêts de la haute Maurienne et de la Tarentaise.

Il a élé préparé jusqu'aujourd'hui, dans ces deux établissements (récolte de 1905 non comprise), les qualités de graineș en kiloıgrammes :

$\begin{array}{lrr}\text { Epicéa } \ldots \ldots \ldots \ldots \ldots \ldots \ldots & 14.152 \\ \text { Mélèze } \ldots \ldots \ldots \ldots \ldots \ldots \ldots & 1.053 \\ \text { Pin Sylvestre } \ldots \ldots \ldots \ldots & 8.679 \\ \text { Pin de montagne........ } & 5.042 \\ \text { Arolle } \ldots \ldots \ldots \ldots \ldots \ldots & 98\end{array}$

Une partie seulement de cesı graines a été ulilisée en Savoie ; le surplus a été r'éparti dans le reste de la France.

En Savoic, les graines résineuses ont été surtout utilisées dans des pépinières. Cependant, aux grandes altitudes et aux expositions froides, là où la sécheresse n'est pas à craindre, on a eu recours au semis. Comme en montagne, à cause de la raideur" des pentes et de l'instabilité des verElant's, il n'est pas possible, comme en plaine, de donner une culture au sol, les semis ont été faits par potets. 298 hectares ont étú ensemencés en Maurlienne, en Tarentaise et dans le Haut Faucigny, qui ont exigé 2.526 kilogriammes de graines de Mélèze, 4.375 kilogrammes de Pin Cembro $\in$ t 20 kilogrammes de Pin Sylvestre.

Mais le mode de reboisement le plus ordinaire, $\epsilon$ st la plantation. Au lieu d'avoir une grande pépinière capable l'alimenter' de plants loute une vallée, le service forestier a crréé, à côté des surfaces à restaur'er, de petites pépinières locales qui permettent d'élever sur place, dans la station même où ils doivent vivre, tous les sujets destinés à être plantés. Ce procédé a l'avantage de supprimer la majeure partic des frais de transport et les risques d'échauffement ou de dessication des plantules.

Comme avec les résineux, la réussite est d'autant plus assurée que les plants sont moins âgés, les plantations ne se font qu'arec des brins très jeunes ayant de $0 \mathrm{~m}$. 10 à $0 \mathrm{~m}$. 12. Suivant les essences, la situation et le terrain de la pépinière, les plants atteignent cette dimension à deux ou trois ans.

De même que le semis, la plantation se fait par" potets ; dans chaque trou on installe une toulfe de deux plants. Par" hectar"e, le nombre des potets est de 7.000 , ce qui correspond à un écartement de 1 m. 20.

Pour déterminer. le choix des espèces ligneuses à employer il faut examiner : $1^{\circ} \mathrm{La}$ base géologique, la fraîcheur et la profondeur du sol ; $2^{\circ}$ L'exposition ; $3^{\circ}$ Les altitudes extrêmes de la surface à traiter ${ }^{*} ; 4^{\circ} \mathrm{La}$ composition des peuplements forestiers les plus voisins.

Dans les régions montagneuse comme la Savoie, l'altitude est un des facteur's les plus importants ; dans le bas, on trouve un climat tempéré auquel sucicède le climat subalpin. Au-dessus règne le climal alpiestre ou alpin.

Lies lignes de démarcation entre ces diverses zones varient suivant les vallées et souvent dans la même vallée.

Voici les essencies qui conviennent le mieux, en Savoie, à chacune de ces divisions :

Zone tempérée. - Saules, Peupliers, Aunes blancs et glulineux, Eirlable, Sycomore, Frêne, Chène rouvre, Hêtre, Robinier, flaux Acacia, Alisieris, Sorbiers, Pin noir d'Autriclíe, Pin Sylvestre, Epicéa.

Zone subalpine. - Saules, Peuplier, Tremble, Aune blanc, Frêne, Sorbier des oiseleurs, Erable plane, Pin Sylvestre, Pin de monitagne, Sapin, Epiciéa, Mélèze.

Zone alpestre - Epicéa, Mélèze, Pin Cembro ou Arolle.

Cie sont encore les conditions locales qui déterminent l'époque à adopter pour exécuter les plantations. D'ordinaire, le printemps est la saison la plus favorable; après la fonte des neiges', le sol; imbibé d'humidité, permet aux jeunes sujets d'entrer immédiatement en végétation, de développer leur enracinement et, partant, de mieux résister au soulèvement produit par le gel pendant l'hiver suivant.

Dans certains cas, cependant, il faut recourir aux plantations d'automne; c'est quand' on se trouve avoir à reboiser un terrain sec, filtrant, exposé au sud et à l'Ouest. Le Mélèze, qui fait éclater ses bourgeons aussitôt que disparaît la neig'e, ne peut guère êtrle planté qu'après l'arrêt de la végétation.

En Savoie, les travalux de reboisement sont relativement peu avancés ; l'Eitat se trouve actuellement, dans les deux départements, dans l'obligation de consolider, à l'aide d'ouvrages diart, les berge's des tor'rents. Ce n'est donc qu'après avoir raffermi les versants qu'il lui sera possible de procéder à l'installation des grrandes espèces ligneuses.

La situation, au point de vue de la restauration des terrains en montagne, est aujour'd'hui la suivante :

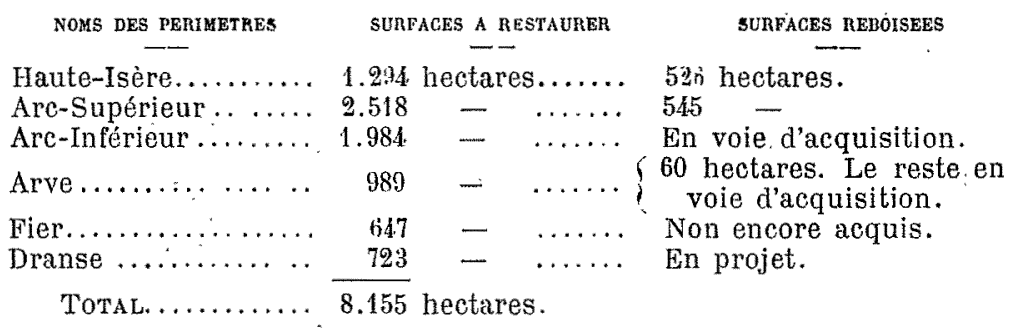

Il est à noter qu'une partie de ces 8.155 hectares est constituée par' des rocis à pic, nus et stériles et qu'une autr'c portion se trouve ani-desisus de la limite supérieure de la végétation forestière. Si l'on compare ce chiffre de 8.155 hectares à la superficie tolale de la Savoie, soit 1.078 .400 hectares, on voil que la surface à restaurer' ne formera que les 7.56 p. 1000 dee l'ensemble des deux départements. Quand on aur'a pu reboiser ces 8.155 hecitares, on sera loin d'avoir pu compenser les défrichements faits diepuis cent vingt ans qui dépassient, pour les forêts communales de la Maurienne et de la Tar'entaise seulement, plus de 16.000 hectares, et encore moins les destructions énormas de forêts qui s'exé- 
cutent aujourd'hui sous nos yeux, déchaùnent les avalanches, engendrent des torrents, des laves et rendent toujour's plus irrég"ulier le débit de nos fleuves, de nos rivières et ont, par suite, sur le r'égime du Rihône, la plus désastreuse influence.

L'Eitalt ne saurait donc, à lui seul, suffire à la tâche énorme de restaurer toutes les montagnes. Si, seul, il peut et doit cicatriser les plaiest les plus dangereuses qui menacent de tout engloutir, c'esit aux particulier's et surtout aux communes propriétaires de tant de friches qu'il appartient de créer les vastes massifs forestier's qui seront à la fois la richesse et la sauvegin"de du pays tout entier.

\section{Les Installations Hydro-Électriques de la Société Énergie Électrique du Littoral Méditerranéen}

La France possède, à l'heure actuelle, d'importants réseaux de distribution d'énergie électrique alimentés par la houille blanche. Parmi ces réseaux, nous citerons celui de la Société Grenobluise de Force et Lumière, dont les fils couvrent le département de l'Isère, à la manière d'une vaste toile d'araignée s'étendant jusque dans les départements du Rhône et de la Loire. Nous citerons encore le réseau de la Sociélé méridionale de Transport de force, qui s'étend.sur tout le département de l'Aude. Mais le plus développé de tous ces réseaux est bien certainement celui de la Société Energie Electrique du Littoral Méditerranéen; dont le distingué directeur général est M. C. Cordier, ingénieur' en chef des Ponts et Chaussées, président du Syndicat des. Forces hydrauliques, réseau qui s'étend, à l'heure actuelle, sur plus de quatre départements, le long de la Méditerranée. La houille blanche sur la Côte d'azur !

Le réseau hydro-électrique de la Société Énergie Électrique comprend tout le bassin du Var, ainsi que celui de la Durance, et utilise les eaux qui tombent sur le vaste espace compris entre la frontière italienne, le massif du Pelvoux, le Vercors, le Rhône et la mer. Bien que cette région soit fort déboisée, el subisse les funestes effets dûs à la transhumance, le régime auquel sont soumises les usines de la Société n'est pas trop défavorable. En effet, d'une part, plusieurs rivières prennent leur source dans la haute montagne et possèdent, sous forme de neige ou de glace, des réserves de houille blanche qui, accumulées durant l'hiver, régularisent le débit pendant l'été. D'autre part, l'ensemble du bassin hydrologique est à une altitude modérée, et dans le voisinage immédiat de cette vaste chaudière à évaporation intense qu'est la Méditerranée, de sorte que les précipitations atmosphériques s'y font abondamment en hiver sous forme de pluie, ce qui rend l'étiage d'hiver relativement abondant par rapport aux cours d'eau à régime purement glaciaire du haut Dauphiné et des Savoies. Certaines rivières, comme l'Argens ou la Siagne, ont leur bassin établi tout entier à basse altitude. Dans son ensemble, le régime hydraulique auquel est soumis l'Énergie Électrique est relativement régulier, avec maximum en hiver, ce qui est tout à l'avantage de cette Société, la période de grande consommation ayant précisément lieu pendant la saison froide, surtout pour toute cette région maritime qui s'étend depuis 'Toulon jusqu'à la frontière italienne, et qui constitue la Côte d'azur $\left(^{\star}\right)$.

(") Pour une partie de cette étude, nous avons eu recours à la conférence faite sur ce sujet, à la Société des Ingénieurs civils de France, par M. DE MARCHENA (voir. Bulletin de juillet 1907), ainsi qu'aux articles sur lë même sujet publiés dans l'Eclairage Electrique sous la signature de M. Friller:
Comme on va le voir par la suite, les installations de l'Énergie Electrique sont un remarquable exemple de l'emploi judicieux de stations centrales à vapeur de secours, placées dans le voisinage immédiat des lieux de consommation.

En outre de l'Énergie Electrique, nous dirons aussi quelques mots d'une de ses filiales, le Srud Electrique.

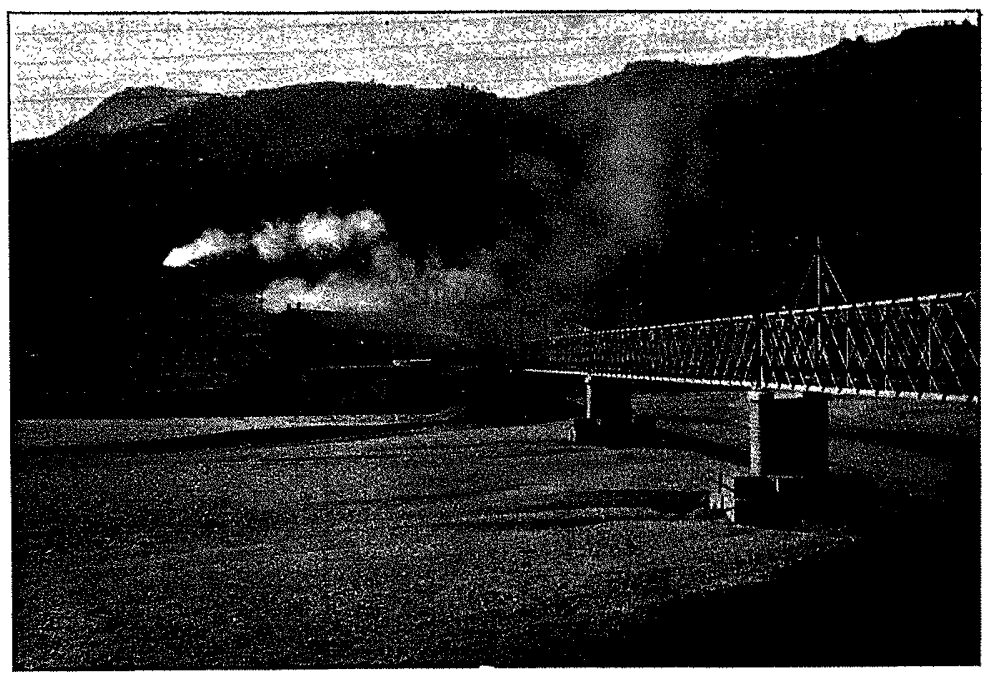

FıG. 1. - Vue de lusine génératrice du Plan-rla-Var el de la fabrique de Carbure de Galcium de la Société Niçoise d'Electrochimic

Historique. - En 1898-1899, la Compagnie des Tramways de Nice et du Littoral, filiale de la Compagnie française Thomson-Houston, coupait, au moyen d'un canal souterrain, la boucle que le Var forme à «la Mescla», aussitôt après son confluent avec la Tinée, et, au moyen d'une dérivation de 600 mètres créait, à 30 kilomètres de Nice, une chute de 10 mètres de hauteur, d'une puissance de 2000 chevaux environ. A la même époque, la Compagnie des Grands Travaux de Marseille étudiait un projet grandiose, celui de l'application de l'électricité à la traction des trains entre Cannes et Vintimille, et s'assurait les droils de riveraineté pour la création d'une chute de $3000 \mathrm{HP}$ sur la rivière le Loup. Le transport d'énergie de la Mescla ayant donné d'excellents résultats, les deux grandes Sociétés précitées décidèrent, en juin 1900, d'associer leurs efforts pour utiliser les ressources hydrauliques de la Provence, au moyen de transports d'énergie à grandes distances, et en faire bénéficier, non seulement les localités voisines, mais encore celles de la côte méditerranéenne, et, à cet effet, elles fon-

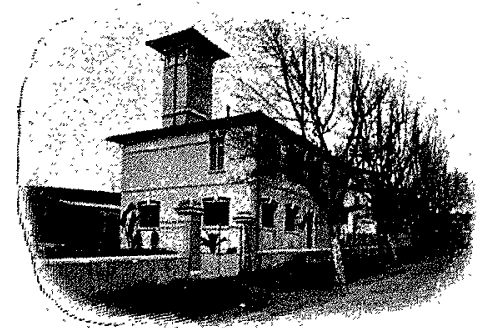

Fig. 2. - Sous station dAntibes. dèrent la Socièté Énergie Électrique du Littoral Mé. diterranéen.

Le programme que cette Sociélé s'est appliquée à réaliser est le suivant :

10 Transporter et distribuer l'énergie électrique, produite par des usines hy dro-électriques, dans toute la région comprise entro le Rhône, le Dauphiné, les Alpes et la Méditerranée

2o Relier entre elles les usines hydro-électriques établies sur des cours d'eau de régimes différents, de manière à équilibrer ces régimes, et à diminuer l’influence des éliages qui se produisent généralement en hiver sur les rivières d'origine glacière (Var, Durance), et en été sur les autres rivières (Loup, Siagne, Verdon, etc.).

$3^{\circ}$ Disposer ses réseaux de manière à assurer une grande sécurité d'exploitation, et à permettre de répartir', suivant les besoins, la charge entre les diverses usines genéra- 